\title{
Application of Blind Deblurring Algorithm for Iris Biometric
}

\author{
F.Alaoui \\ Faculty of science \\ Chouaib doukkali El- \\ jadida
}

\author{
K.Assid \\ Faculty of science \\ Chouaib doukkali El- \\ jadida
}

\author{
V.Dembele \\ Faculty of science \\ Chouaib doukkali El- \\ jadida
}

\author{
A.Nassim \\ Faculty of science \\ Chouaib doukkali El- \\ jadida
}

\begin{abstract}
Iris recognition is a form of biometric technology that authenticates individuals by using the unique iris patterns between the pupil and the sclera. There are three factors: Defocus, Motion Blur, and Off-Angle to substantially degrade performance more than the other quality. The work described in this paper is interested in Motion Blur. The iris image will appear blurry which can reduce iris recognition accuracy. The focus of the article is to achieve a quality edge preserving image restoration using Total Variation (TV)-L1 regularization technique. L1 norm based approaches do not penalize edges or high frequency contents in the restored image.
\end{abstract}

Experimental results showed that the iris recognition accuracy was better than that when using debluring algorithms.

This article presents two contributions over previous research. (1) A new application to deblurring iris image using fast TV11 deconvolution model is proposed. (2) Previous research restored coexisting motion blurred images in terms of visibility, but in this article, we restored them in terms of recognition

\section{General Terms}

Pattern Recognition, Biometrics.

\section{Keywords}

Iris Biometric, Motion blur, Deconvolution.

\section{INTRODUCTION}

Biometric has been used to identify an individual person based on some sort of unique patterns. Biometrics systems can use one or more of several different physical and/or behavioral characteristics for identification (one-to-many) and verification (one-to-one) such as fingerprints [1], palm print [2], and the one presented in this paper the iris [3-4-5]. The patterns is almost impossible to be imitated by others and not be easily changed by natural causes, e.g. temperature, climate, age, illness, etc. The uniqueness of the pattern is one advantages of biometrics.

Iris recognition has received increasing attention in recent years because the patterns within the iris are very unique for each person, and even the left eye is also different from the right eye iris [6]. Iris patterns are more stable, unique and noninvasive and will provide a promising solution to high security. Those iris recognition approaches can be divided into six categories according to various iris features: phasebased methods [4- 7- 8], texture analysis [9- 10- 11- 12- 13], zero-crossing representation [14- 15- 16- 17], intensity variation analysis [18- 19], fractal dimension analysis [20] and neural network [21].
Daugman's algorithms [22] use the 2D Gabor wavelet transform (phase based method) in order to extract the spatial frequency. The result is a set of complex numbers that carry local amplitude and phase information for the iris image, all amplitude information is discarded, and the resulting 1024 bits (Iris code) that represent an iris consist only of the complex sign bits of the Gabor-domain representation of the iris image. Matching algorithm calculates hamming distance between query iris image and image in the database (The CASIA iris database was used in this study to evaluate the performance of the proposed method), producing authentication based on minimum value of it.

The algorithms developed by Daugman have good recognition performance, but this may have been achieved under favorable conditions. Motion blurring can significantly reduce iris recognition accuracy.

In this paper, a new application is proposed to deblurring iris image using fast TV-11 deconvolution model [23]. The algorithm is fast and accurate to restore the clear image. This paper is organized as follows:

In section 2 we present the image quality assessment for iris biometric in terms of motion blur and a new method is proposed to restore (blind deconvolution) iris image; finally the experimental results and conclusion are show in section 3 and 4.

\section{IMAGE QUALITY ASSESSMENT}

\subsection{Motion Blur Assessment Method}

Image quality assessment plays an important role in automated biometric systems for two reasons: (i) system performance (recognition and segmentation), and (ii) interoperability. In this paper we assess image quality from the iris biometric. There are three factors [24]: Defocus, Motion Blur, and Off-Angle to substantially degrade performance more than the other quality. The work described in this paper is interested in Motion Blur.

The blurring process is modeled as the following convolution:

$$
\mathrm{I}(\mathrm{x}, \mathrm{y})=(M \circledast P)(x, y)+n(x, y)
$$

Where $\circledast$ is the convolution operator, $\mathrm{M}$ is the original image, $\mathrm{I}$ is the degraded image and $\mathrm{P}$ is the blurring kernel (Point Spread Function), and $\mathrm{n}$ is the noise.

The motion-blur kernel is very different from the kernels of other types of blurring (e.g. out-of-focus blurring, Gaussiantype optical blurring), as there exist not simple parametric forms to represent motion-blur kernels. 
The Blur kernel can be presented on function the two parameters $\mathrm{C}$ and $\mathrm{v}(\mathrm{x}, \mathrm{y})$, where $\mathrm{C}$ is a continuous curve of finite length in $R_{2}$ which denotes the camera trajectory and $\mathrm{v}(\mathrm{x}, \mathrm{y})$ is the speed function which varies along $\mathrm{C}$.

In general, recovering the latent image $M$ from the blurry observation $\mathrm{I}$ is a deconvolution problem, which is further separated into the non-blind and blind cases. In the non-blind case, the blur kernel P is assumed to be known or estimated, and the task is to recover the clear image $\mathrm{M}$ by reversing the effect of convolution on the blurred image I. There have been extensive studies on robust non-blind deconvolution algorithms [25- 26- 27- 28- 29]. In the case of blind deconvolution problem, both the blur kernel $\mathrm{P}$ and the latent image $\mathrm{M}$ are unknown, which is a more challenging task. Motion deblurring is a typical blind deconvolution problem, because the motion between the camera and the scene always varies for different images.

The blind deconvolution is formulated as a joint minimization problem with regularizations:

$$
M^{*}, P^{*}=\|M \circledast P-I\|_{2}+\Theta_{P}(P)+\Theta_{M}(M)(2.2)
$$

Here $\|\mathrm{M} \circledast \mathrm{P}-\mathrm{I}\|_{2}$ is the data fitting term. To overcome the inherent ambiguities between the blur kernel $\mathrm{P}$ and the clear image $\mathrm{M}$, certain regularization terms on both $\mathrm{P}$ and $\mathrm{M}$ have to be added in the minimization $\left(\Theta_{P}(P)\right.$ and $\Theta_{M}(M)$ are the regularization terms on the blur kernel and the latent image). In this section, a two phase method is introduced for PSF estimation. The first stage aims compute a coarse version of the kernel. In the second phase non-convex optimization is employed.

\subsection{Sparse Kernel Estimation.}

\subsubsection{Salient edges prediction}

The work in [23] has discussed the usefulness of edge information and demonstrated that the edges of large-scale objects are significant to the kernel estimation. The sharp edges are firstly selected using the blurred image. For each pixel $\mathrm{i}$ in the blurred image $\mathrm{I}_{\mathrm{i}}$, we form a local window $\mathrm{W}_{\mathrm{h}(\mathrm{i})}$ centered at it with size $h * h$, which is the same as that of the corresponding blur kernel at scale 1 . The usefulness of the edges is measured as:

$$
r(i)=\frac{\left\|\sum_{j \in w_{h}(i)} \nabla I(j)\right\|_{2}}{\sum_{j \in w_{h}(i)}\|\nabla I(j)\|_{2}+\kappa} \quad(\mathbf{2 . 3})
$$

Where $\nabla \mathrm{I}=\left(\partial_{\mathrm{x}} \mathrm{I}, \partial_{\mathrm{y}} \mathrm{I}\right)$ is the image gradient. A large value $\mathrm{r}$ implies that the image structure is strong in the local window. When the local region is flat or has fine texture, the gradients will be neutralized by each other, so that the value of $\left\|\sum_{\mathrm{j} \in \mathrm{w}_{\mathrm{h}}(\mathrm{i})} \nabla \mathrm{I}(\mathrm{j})\right\|$ is small. $\kappa$ serves to prevent producing a large $r$ in flat regions. We construct a matrix $L_{r}$ as a binary mask using a unit step function

$$
\mathrm{L}_{r}(\mathrm{i})=\eta_{\tau_{r}}\left(r(i)-\tau_{r}\right)= \begin{cases}0, & r(i)<\tau_{r} \\ 1, & r(i) \geq \tau_{r}\end{cases}
$$

For each pixel, $L_{r}(i)=1$ if the $r$-value is larger than the threshold $\tau_{r}$, and $L_{r}(i)=0$ is otherwise.
The further selection of sharp edges is determined by the gradient magnitude of shock filtered image. Shock filter [30] is an effective tool to restore strong edges from the blurred signals. In $\mathrm{M}_{\mathrm{t}}$ denote the Shock filter image recovered from the blurred image. The final selected edges for kernel estimation are determined as:

$$
\begin{aligned}
& \nabla M^{s}=\nabla M_{\mathrm{t}} \circ L_{s} \\
& L_{S}(i)=\eta_{\tau_{S}}\left(\left(\left\|\nabla M_{t}\right\|_{2} \circ L_{r}\right)-\tau_{S}\right)
\end{aligned}
$$

Where $\circ$ is the element-wise multiplication operator.

\subsubsection{Estimating the blur kernel}

The initial kernel estimation is obtained by iteratively performing the salient edges selection, kernel estimation and image deconvolution at each level 1 . Based on the salient sharp edges $\nabla M_{1}^{S}$; we rewrite the function with a Gaussian regularized term as:

$$
\widehat{P^{\mathrm{l}}}=\left\|\nabla M_{l}^{S} \circledast P-\nabla I^{l}\right\|_{2}+\lambda_{P}\|\mathrm{P}\|_{2} \quad(2.6)
$$

Where $\lambda_{\mathrm{P}}$ is a weight and the regularizer $\|\mathrm{P}\|_{2}$ is similar to [23- 31- 32]. Eq. (2.6) can be calculated efficiently in the Fourier domain by:

$$
\widehat{P}^{\mathrm{l}}=\mathcal{F}^{-1}\left(\frac{\overline{\mathcal{F}\left(\partial_{\mathrm{x}} \mathrm{M}^{\mathrm{s}}\right)} \mathcal{F}\left(\partial_{\mathrm{x}} \mathrm{I}^{\mathrm{l}}\right)+\overline{\mathcal{F}\left(\partial_{\mathrm{y}} \mathrm{M}^{\mathrm{s}}\right)} \mathcal{F}\left(\partial_{\mathrm{y}} \mathrm{I}^{\mathrm{l}}\right)}{\mathcal{F}\left(\partial_{\mathrm{x}} \mathrm{M}^{\mathrm{s}}\right)^{2}+\mathcal{F}\left(\partial_{\mathrm{y}} \mathrm{M}^{\mathrm{s}}\right)^{2}+\lambda_{\mathrm{k}}}\right)
$$

Where $\mathcal{F}(\cdot)$ and $\mathcal{F}^{-1}(\cdot)$ represent the FFT and inverse FFT respectively. $\overline{\mathcal{F}(.)}$ is the complex conjugate operator.

\subsubsection{Corse image estimation}

In the image deconvolution, we use the sharp edge gradient $\nabla M^{S}$ as a spatial prior in order to guide the recovery of a coarse version of the latent image. We rewrite the function as:

$$
\widehat{M^{1}}=\left\|M \circledast \widehat{P^{1}}-\mathrm{I}^{1}\right\|_{2}+\lambda_{M}\left\|\nabla M-\nabla M_{l}^{S}\right\|_{2}
$$

Where $\lambda_{M}$ is the regularization Weight. The frequency domain the solution of Eq. (2.8) can be expressed as:

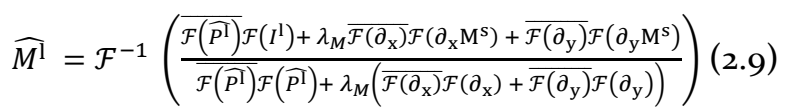

\subsubsection{ISD-Based Kernel Refinement}

To enhancing the sparsity of the kernel, the ISD method is applied to refine it. In [33], the implementation of ISD, called threshold-ISD, is as fast as L1-minimization but requires fewer measurements. The algorithm forms a partial support $\mathrm{S}=\{\mathrm{j} \mid \mathrm{P}(\mathrm{j})>\tau\}$ based on the current estimation. The regularization term is replaced in Eq. (2.6) by $\sum_{\mathrm{j} \epsilon \overline{\mathrm{S}}}|\mathrm{P}(\mathrm{j})|$ and get:

$$
\widetilde{\mathrm{P}}=\left\|\nabla \mathrm{M}_{1}^{\mathrm{s}} \circledast \mathrm{P}-\nabla \mathrm{I}^{\mathrm{l}}\right\|_{2}+\beta \sum_{\mathrm{j} \epsilon \overline{\mathrm{S}}} \mathrm{P}(\mathrm{j}) \mid(2.10)
$$


Then the large-value elements of kernel are preserved, while the insignificant elements are iteratively suppressed to reduce the noise. Interested readers can refer to [33] for more details.

\subsection{Fast TV 11 Deconvolution.}

Given the estimation $\widetilde{\mathbf{P}}$ of the kernel, to compute the final deblurred result, a TV 11 model is proposed in deconvolution, which is written as:

$$
\widetilde{\mathrm{M}}=\|\mathrm{M} \circledast \widetilde{\mathrm{P}}-\mathrm{I}\|_{2}+\gamma\|\nabla \mathrm{M}\|_{1}(2.11)
$$

The deblurred image is recovered by the deconvolution technique proposed in [34]. The final result shown the small edges and textures are restored well and few artifacts are introduced.

\section{EXPERIMENTAL RESULTS}

There are many standard iris databases that could be employed in iris researches [35]. The CASIA iris database was used in this study to evaluate the performance of the proposed method, but the database does not include many motion blurred iris images. Therefore, the motion blurred iris images was artificially produced using MATLAB (Fig 3.1).

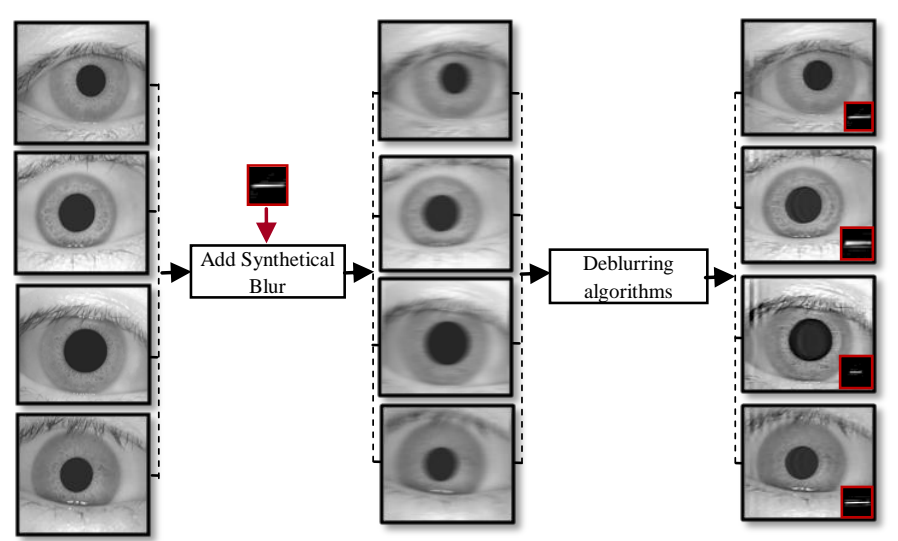

Fig 3.1: (a) The left image shows the input Iris images (CASIA v1); (b) the Middle images show the blurring result. (c)The Right images shows the deblurring iris images and Motion blur kernel

The Chinese Academy of Sciences - Institute of Automation (CASIA) eye image database contains 756 grayscale eye images with 108 unique eyes or classes and 7 different images of each unique eye. Experimentation was carried out on total of 30 iris images with 10 different classes where each class contains 3 images (Left, Right and Centre).

In the experiment [Fig 3.1 (a), Fig 3.1 (c)], we artificially produced motion blurred iris images from 30 persons, the synthetic blurry images were generated by convolving the iris images with a $15 \times 15$ synthetic kernel to authenticate via verification (one-to-one template matching). A template created by imaging the iris, is compared to a stored value template in a database (fig 3.2).

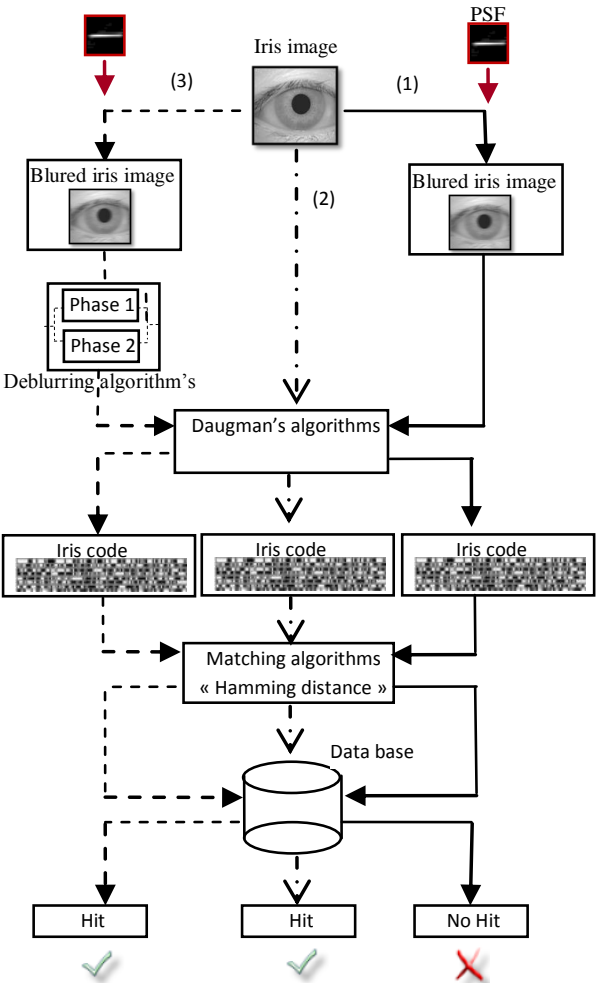

Fig 3.2: (1) Authenticate blurred iris image with synthetic blur (2) Authenticate iris image without Blur (3) Authenticate iris image (first the image is blurred with synthetic blur and deblurred)

The motion blur can be in the form of a translation, a rotation, or some combinations of these. It's characterized by the camera trajectory $(\mathrm{C})$ and the speed function $v(x, y)$ which varies along $\mathrm{C}$. in this work only the important case of a global translation and variations of $v(x, y)$ will be considered (Fig 3.3).

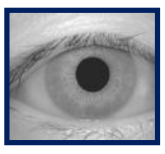

$\mathrm{C}=1, \mathrm{~V}=1$
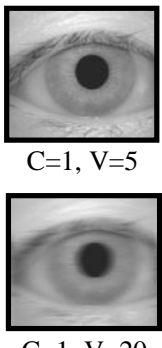
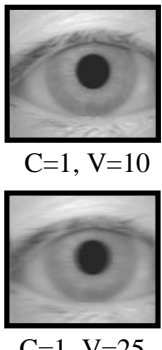

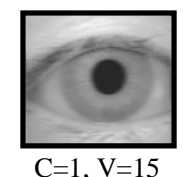

$\mathrm{C}=1, \mathrm{~V}=15$
Fig 3.3: Examples of motion blurred iris images with different values of speed function $v(x, y)$

The proposed deblurring algorithm is based on a mathematical idea introduced in [23].This method restored blurred images in terms of visibility, but we restored them in terms of recognition, generally allows using the default or automatically adapted parameter values. Matlab implementation spends about 0.82 seconds to estimate a debluring image from a $320 \times 280$ iris image (Gray scale) with an Intel Core2Quad CPU Q8400@ 2.66G.

The result of iris authentication was determined by the HD (Hamming Distance) between the enrolled iris codes and the iris codes which were extracted from the input iris images. Users with the input iris were accepted as enrolled users when 
the HD was less than the predefined threshold (0.30). Users were rejected as un-enrolled users when the HD was greater than the threshold.

To compare the change of hamming distance by motion blurring, we measured the difference between iris image without and with motion blurring. If $v(x, y)$ varied between 1 and 21 , the user was accepted and the iris recognition has a good accuracy without using the deblurring algorithm, but if $v(x, y)$ is greater than $\mathrm{c}=21$ the FRR (False Rejection Rate) is increase (The FRR refers to an error that rejects the genuine person enrolled in the recognition system) to overcome this problem, the proposed deblurring algorithm is used to restore the motion-blurred iris images. The results are shown in Table 3.1 and table 3.2.

Table 3.1. The accuracy of iris recognition

\begin{tabular}{|c|c|}
\hline $\begin{array}{c}\text { Motion Blur } \\
\text { Image \# }\end{array}$ & $\begin{array}{c}\text { Hamming Distance (Image Réf*/ } \\
\text { Image \#) }\end{array}$ \\
\hline $\mathrm{C}=1, \mathrm{v}=1$ & 0 \\
\hline $\mathrm{C}=1, \mathrm{v}=2$ & 0.0625756 \\
\hline $\mathrm{C}=1, \mathrm{v}=3$ & 0.0901391 \\
\hline $\mathrm{C}=1, \mathrm{v}=4$ & 0.106267 \\
\hline $\mathrm{C}=1, \mathrm{v}=5$ & 0.124356 \\
\hline $\mathrm{C}=1, \mathrm{v}=6$ & 0.134183 \\
\hline $\mathrm{C}=1, \mathrm{v}=7$ & 0.182692 \\
\hline $\mathrm{C}=1, \mathrm{v}=8$ & 0.155603 \\
\hline $\mathrm{C}=1, \mathrm{v}=9$ & 0.192437 \\
\hline $\mathrm{C}=1, \mathrm{v}=10$ & 0.203619 \\
\hline $\mathrm{C}=1, \mathrm{v}=11$ & 0.209242 \\
\hline $\mathrm{C}=1, \mathrm{v}=12$ & 0.216328 \\
\hline $\mathrm{C}=1, \mathrm{v}=13$ & 0.223168 \\
\hline $\mathrm{C}=1, \mathrm{v}=14$ & 0.246402 \\
\hline $\mathrm{C}=1, \mathrm{v}=15$ & 0.264124 \\
\hline $\mathrm{C}=1, \mathrm{v}=16$ & 0.268553 \\
\hline $\mathrm{C}=1, \mathrm{v}=17$ & 0.271613 \\
\hline $\mathrm{C}=1, \mathrm{v}=18$ & 0.275472 \\
\hline $\mathrm{C}=1, \mathrm{v}=19$ & 0.283081 \\
\hline $\mathrm{C}=1, \mathrm{v}=20$ & 0.297529 \\
\hline $\mathrm{C}=1, \mathrm{v}=21$ & $\mathbf{0 . 3 5 8 5 2 9}$ \\
\hline
\end{tabular}

*Image Réf: image with parameter $(\mathrm{c}=1, \mathrm{v}=1)$

Table 3.2. The accuracy of iris recognition (With Motion Blur and After Deblurring)

\begin{tabular}{|c|c|c|}
\hline \multirow[t]{2}{*}{ Motion Blur Image \# } & \multicolumn{2}{|c|}{ Hamming Distance (HD) } \\
\hline & $\begin{array}{l}\text { With } \\
\text { Motion } \\
\text { Blur }\end{array}$ & $\begin{array}{c}\text { After } \\
\text { Deblurring }\end{array}$ \\
\hline $\mathrm{C}=1, \mathrm{v}=22$ & 0.415954 & 0.243769 \\
\hline$\ldots$ & & \\
\hline $\mathrm{C}=1, \mathrm{v}=26$ & 0.426267 & 0.262432 \\
\hline $\mathrm{C}=1, \mathrm{v}=27$ & 0.435165 & 0.263931 \\
\hline $\mathrm{C}=1, \mathrm{v}=28$ & 0.435961 & 0.292205 \\
\hline $\mathrm{C}=1, \mathrm{v}=29$ & 0.433959 & 0.267011 \\
\hline $\mathrm{C}=1, \mathrm{v}=30$ & 0.501491 & 0.298189 \\
\hline $\mathrm{C}=1, \mathrm{v}=31$ & 0.445417 & 0.423412 \\
\hline $\mathrm{C}=1, \mathrm{v}=32$ & 0.463562 & 0.446094 \\
\hline $\mathrm{C}=1, \mathrm{v}=33$ & 0.430247 & 0.4144227 \\
\hline
\end{tabular}

As shown in Fig 3.4, we measured the Hamming distance for intra-class with motion blurring and after deblurring, experimental results showed that the FRR was decrease when using the proposed method.

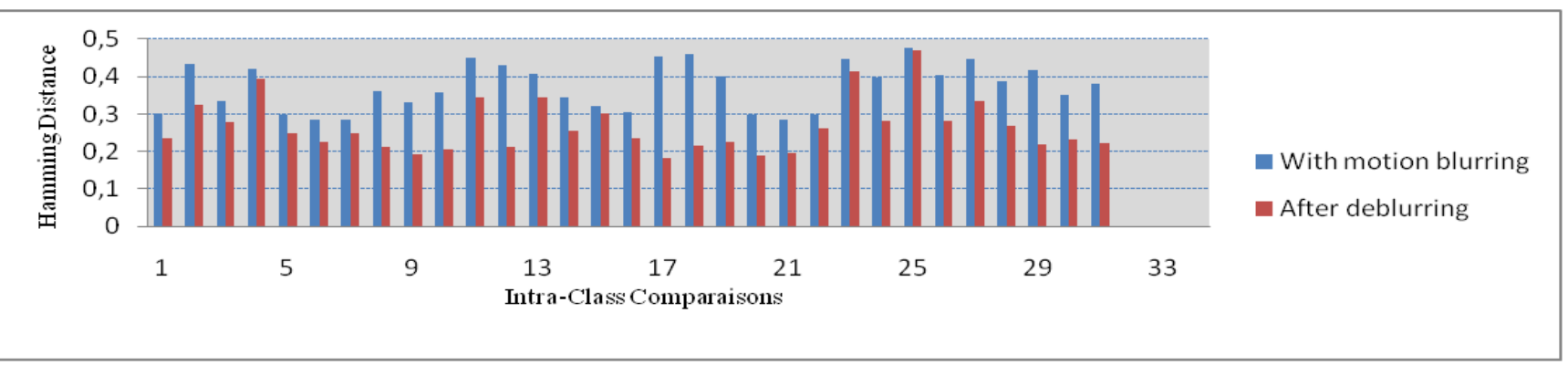

Figure 3.4: Hamming distance distributions, Intra-Class Comparisons (With motion blurring / After deblurring)

\section{CONCLUSIONS}

In this article, a novel restoration method of motion-blurred iris images is proposed. Therefore, iris recognition accuracy was better than that when using debluring Algorithms. However, a suggestion for future work is to study motion restoration method for nonlinear motion blurred (variation of camera trajectory $(\mathrm{C})$ and the speed function $v(x, y))$ iris images.

\section{REFERENCES}

[1] X. P. Luo, J. Jain, "Knowledge based fingerprint image enhancement", Proc.International Conference on Pattern
Recognition (ICPR),Barcelona, Spain, vol. 4,September 2000, pp. 783-786.

[2] D. Zhang, W. K. Kong, J. You and M.Wong, "Online palmprint identification", IEEE Transactions on Pattern Analysis and Machine Intelligence,vol. 25, September 2003, pp. 1041-1050.

[3] P. Kronfeld, "The gross embryology of the Eye", The Eye, Vol.1, 1968, pp.166. 
[4] J. Daugman, "How iris recognition works," IEEE Transactions on Circuits and Systems for Video Technology, Vol. 14, No.1, 2004, pp. 21-30.

[5] L. Flom and A. Safir, "Iris Recognition system"SSS, U.S.Patent: 4, 641, 349, 1987.

[6] Barros, J, French, J and Martin, W.; "Indexing MultiSpectral Images for Content-Based Retrieval", University of Virginia Technical Report, CS-94-40, (1994).

[7] J. Daugman, "Statistical Richness of Visual Phase Information: Updateon Recognizing Persons by Iris Patterns," International Journal on Computer Vision, volume 45(1), 2001, pp. 25-38.

[8] J. Daugman, "Demodulation by complex-valued wavelets for stochastic pattern recognition," International Journal on Wavelets, Multiresolution and Information Processing, Vol. 1, No. 1, 2003, pp. 1-17.

[9] Wildes, R.P., "Iris Recognition: An Emerging Biometric Technology", Proc. Of the IEEE, Vol. 85, No.9, 1997, pp.1348-1363.

[10] Y.Zhu, Tieniu Tan, and Yunhong Wang, "Biometric Personal Identification Based on Iris Patterns", Proceedings of the 15International Conference on Pattern Recognition, Vol. 2, 2000, pp.805 - 808.

[11] S. Lim, K. Lee, O. Byeon, and T. Kim, "Efficient Iris Recognition through Improvement of Feature Vector and Classifier", Journal of Electronics and Telecommunication Research Institute, Vol. 23, No. 2, 2001, pp. $61-70$.

[12] L. Ma, T. Tan, Y. Wang and D. Zhang, "Personal Identification based on Iris Texture Analysis", IEEE Transactions on Pattern Analysis and Machine Intelligence, Vol. 25, No. 12, 2003, pp. 1519-1533.

[13] B.R.Meena, "Personal Identification based on Iris Patterns", Ph.D Thesis, Department of Computer Science and Engineering, Indian Institute of Technology, Kanpur, 2004.

[14] W. W. Boles, and B. Boashash, "A human identification technique using images of the iris and wavelet transform," IEEE Transactions on Signal Processing, volume 46(4), 1998, pp. 1185-1188.

[15] C. Sanchez-Avila, and R. Sanchez-Reillo, "Multiscale Analysis for Iris Biometrics", Proceedings of 36th International Conference on Security Technology, Vol. 1, 2002, pp 35-38.

[16] S.Avila, and Sanchez-Reillo R, "Two different approaches for iris recognition using Gabor filters and multiscale zero-crossing representation", Pattern Recognition, Vol: 38, 2005, pp: 231-240.

[17] M.Monro, Soumyadip Rakshit, and Dexin Zhang, "DCTBased Iris Recognition", IEEE Transactions on Pattern analysis and Machine Intelligence, Vol.29, No.4, 2007, pp.586-595.

[18] Y.Ping Huang, Si-Weiluo, and En-Yi Chen, "An Efficient Iris Recognition system", Proceedings of the First International conference on Machine Learning and Cybernatics, Beijing, November 2002, pp. 450-454.
[19] L. Ma, T. Tan, Y. Wang, and D. Zhang, "Local intensity variation analysis for iris recognition," Pattern Recognition, volume 37(6), 2004, pp. 1287-1298.

[20] W.Shiung Chen, and Shang-Yuan Yuan, "A Novel Personal Biometric Authentication Technique using Human Iris Based on Fractal Dimension features", Proceedings of ICASSP, Vol.3, 2003, pp. 201-204.

[21] L.Liam, Ali Chekima, Liau Fan, and Jamal Dargham, "Iris recognition using self-organizing neural network", Proceedings of IEEE Student Conference on Research and Developing Systems', 2002, pp.169-172.

[22] J. Daugman, "Biometric Personal Identification System Based on Iris Analysis," US patent 5291560, Patent and Trademark Office, Washington, D.C.1994.

[23] L.Xu and Jiaya Jia, "Two-Phase Kernel Estimation for Robust Motion Deblurring", European Conference on Computer Vision (ECCV), 2010.

[24] N. D. Kalka, V. Dorairaj, Y. N. Shah, N. A. Schmid, B. Cukic, "Image Quality Assessment for Iris Biometric" Lane Department of Computer Science and Electrical Engineering West Virginia University, Morgantown, WV-26506, USA

[25] H. C. Andrews, B. R. Hunt, Digital image restoration, Prentice-Hall, En-glewood Cliffs, NJ, 197.

[26] M. K. Ng, R. H. Chan, W. Tang, A fast algorithm for deblurring models with neumann boundary condition, SIAM J. Sci. Comput. 21 (3) (2000) 851-866.

[27] T. F. Chan, J. Shen, Image processing and analysis, Variational, PDE, wavelet, and stochastic methods, Society for Industrial and Applied Mathematics (SIAM), Philadelphia, PA, 2005.

[28] A. Chai, Z. Shen, Deconvlolution: A wavelet frame approach, Numer. Math.106 (2007) 529-587.

[29] Y. Lou, X. Zhang, S. Osher, A. Bertozzi, Image recovery via nonlocal operators, UCLA CAM Reports (08-35).

[30] S. Osher and L. I. Rudin. Feature-oriented image enhancement using shock filters. SIAM Journal on Numerical Analysis, 1990, 27(4):919-940.

[31] S. Cho and S. Lee. Fast motion deblurring. ACM Transactions on Graphics (Proc. SIGGRAPH Asia) , 2009, 28(5):1-8.

[32] L. Yuan, J. Sun, L. Quan, and H. Shum. Image deblurring with blurred/noisy image pairs. ACM Transactions on Graphics (Proc. SIGGRAPH), 2007, 26(3):1-10.

[33] Y. Wang and W. Yin. Sparse signal reconstruction via iterative support detection. SIAM Journal on Imaging Sciences, 2010, 3(3):462-491.

[34] Y. Wang, J. Yang, W. Yin, and Y. Zhang. A new alternating minimization algorithm for total variation image reconstruction SIAM Journal on Imaging Sciences, 2008, 1(3):248-272.

[35] CASIA Iris Image Database (ver. 1.0), http://www.sinobiometrics.com/casiairis.html. 